\title{
Factores asociados a riesgo de infección bacteriana grave en niños bajo 24 meses de edad, internados por fiebre sin foco aparente
}

\author{
Carolina Torregrosa, Cristian García R, Juan Sciarotta, Carlos Vay, Silvia Caíno, y Alejandro Ellis
}

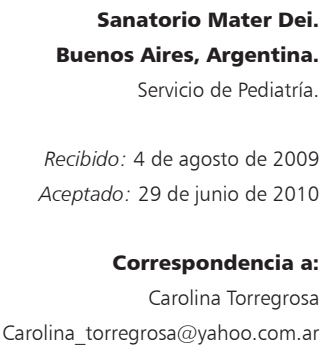

\section{Associated risk factors for serious bacterial illness in children less than 24 months, admitted with fever without source}

Objectives: To analyze risk factors for serious bacterial illness (SBI) in children less than 2 years of age admitted with fever without source. Population: Children under 2 years admitted at the Pediatric Service of Sanatorio Mater Dei from May 2004 to December 2005. Design: Observational, prospective and longitudinal study. Materials and Methods: Independent variables: age, gender, siblings, household viral infections, day care attendance, vaccination, season, laboratory data, Rochester criteria, YIOS scale, antibacterial treatment, length of hospitalization, and final diagnosis. A multiple logistic regression stepwise model was used. Results: 70 out of 201 patients developed SBI, 56 were under 3 months of age. Predominant diagnosis were: urinary tract infection (n: 47), meningitis (n: 11) and bacteremia (n: 9). Predictive factors: white-cells count $>15.000 / \mathrm{mm}^{3}(\operatorname{coef} 1.05$, $\mathrm{OR}=2.17,95 \%$ CI 1.13-4.15) and household viral contact (coef -0.79, OR 0.42, CI 0.23-0.77). Conclusions: Risk factor for SBI was leucocytes count $>15.000 / \mathrm{mm}^{3}$. Household viral contact proved being a protective factor.

Key words: Fever, febrile infant, serious bacterial illness.

Palabras clave: Fiebre, lactante febril, infección bacteriana grave.

\section{Introducción}

L a fiebre en el lactante es motivo de consulta frecuente en la práctica pediátrica. La mayoría de los autores consideran a los lactantes de 90 días o menores como un grupo de especial riesgo para la adquisición de infecciones bacterianas graves (IBG), si bien otras publicaciones realizan este corte a los 60 días de vida $^{1-5}$. Desde el punto de vista fisiológico y desarrollo del sistema inmunológico, cualquier intento de poner un punto de corte a determinada edad resulta artificial, ya que ningún cambio abrupto indica una clara transición de un sistema inmunológico parcialmente inmaduro a otro más eficaz. Desde la epidemiología, la bacteriología y la clínica es posible encontrar, quizás, un mayor sustento. La elevada incidencia de infecciones graves en los neonatos $^{6,7}$, comparados con lactantes entre 30 y 90 días, le da a este grupo un carácter singular. La mayor frecuencia de ciertos agentes como Listeria sp y Enterococcus sp en los pacientes menores de 60 días de vida pone entonces, hacia el final de la $8^{\mathrm{a}}$ semana un punto de corte con respecto a lactantes con mayor edad ${ }^{6}$. La poca confiabilidad que ofrecen los signos y síntomas en el grupo de neonatos y lactantes bajo 90 días de edad marca una nueva distinción.

La mayoría de los niños febriles concurrentes al con- sultorio y/o departamentos de urgencias tiene menos de 24 a 36 meses de edad; esto hace que el enfoque deba ser lo más claro posible, reconociendo que hay diferencias sustanciales entre el grupo $<60-90$ días con aquel grupo entre 91 días y 24 a 36 meses de edad ${ }^{5,8-10}$. El propósito clínico frente a estos pacientes es diferenciar aquellos que pueden presentar infección grave e IBG del resto de los pacientes. No obstante, y sobre todo sobre los 3 meses de edad y menores de 24 meses, los criterios de selección de internación no siempre son claros y muchos de ellos son internados sólo por presentar algunos parámetros de riesgo.

El objetivo del estudio fue determinar factores de riesgo asociados a IBG en niños bajo 2 años de edad, que fueron internados por fiebre y $\sin$ foco aparente.

\section{Pacientes y Métodos}

Estudio prospectivo, observacional, longitudinal y analítico que incluyó a todos los niños bajo 24 meses de edad, internados en el Servicio de Pediatría del Sanatorio Mater Dei, entre el $1^{\circ}$ de mayo de 2004 y 31 de diciembre de 2005, cuyo motivo de ingreso fue fiebre $\sin$ foco aparente. 
Definiciones. Se denominó fiebre a todo niño cuya temperatura axilar fue $\geq 38{ }^{\circ} \mathrm{C}$. Se consideró fiebre sin foco aparente a todo paciente con fiebre en el que no se pudo establecer la etiología luego de una historia clínica y examen físico detallado.

Las variables estudiadas fueron: edad, sexo, época del año, escala de YIOS al ingreso, presencia de infección viral en el hogar, vacunación previa contra Haemophilus influenzae tipo b, Streptococcus pneumoniae y Neisseria meningitidis, presencia de fenómenos bacteriémicos y signos meníngeos. El estudio solicitado básico incluyó: hemograma y velocidad de eritrosedimentación (VHS), orina completa, radiografía de tórax, hemocultivos y urocultivo. Se registraron además: recuento de leucocitos mayor o menor de $15.000 / \mathrm{mm}^{3}$, VHS mayor o menor de $30 \mathrm{~mm}$ en $1^{\mathrm{a}}$ hora, y recuperación microbiológica en los cultivos. Otros exámenes fueron solicitados según manifestaciones clínicas aparecidas en el curso de la evolución. No se incluyó la determinación de PCR cuantitativa (no disponible como estudio de rutina en ese entonces). La IBG se certificó mediante cultivos positivos, empleando técnicas estándares, de sitios normalmente estériles (sangre, orina y líquido cefalorraquídeo, líquido articular) según correspondiera. Consideramos infección urinaria a aquella con reacción inflamatoria (leucocitos mayores de 10/campo) y cultivo monomicrobiano con recuento $\geq 100.000 \mathrm{ufc} / \mathrm{mm}^{3}$ en una muestra obtenida mediante cateterismo vesical o recolector de orina.

Se registraron días de hospitalización, tratamiento antimicrobiano empírico inicial, diagnóstico de egreso, y sitios de infección con documentación etiológica si la hubiera.

Se definió IBG a todo niño cuyo diagnóstico de egreso fue sepsis, meningitis bacteriana, neumonía, infección urinaria, diarrea invasora (Salmonella spp, Shigella spp, Campylobacter spp, Escherichia coli enteroinvasora), artritis séptica u osteomielitis, con cultivo positivo en sangre, líquido cefalorraquídeo, orina, deposiciones, líquido articular y/o aspiración ósea, o la presencia de dos o más hemocultivos positivos para Staphylococcus coagulasa negativa o Corynebacterium sp.

Se calculó la tasa de IBG y la tasa específica según sexo, edad y época del año.

La asociación de variables categóricas se analizó mediante test chi cuadrado.

Para el análisis de factores de riesgo se dividió a la población en dos grupos según que presentaran o no diagnóstico de egreso de IBG. Las variables que se consideraron como posibles predictoras de IBG fueron: edad ( $<3$ meses, 3-12 meses, $>12$ meses), sexo, número de hermanos, infección viral en el hogar (presencia/ ausencia), concurrencia a jardín maternal (si/no), vacunación previa para $S$. pneumoniae, $H$. infuenzae tipo b, y N. meningitidis grupo C, fiebre (mayor o menor de $39^{\circ}$
C), recuento de leucocitos ( $>$ o $<$ de $\left.15.000 / \mathrm{mm}^{3}\right)$, VHS $\left(>\mathrm{o}<30 \mathrm{~mm}\right.$ en la $1^{\mathrm{a}}$ hora), y escala de YIOS- young infant observation scale (puntajes 0-1) correspondiendo 0 a ausencia de riesgo de IBG. Para la evaluación de alto o bajo riesgo se utilizó principalmente los criterios de Rochester, pero esto último no fue realizado como criterio de selección para internación sino que fue medido en el paciente ya internado.

Se calculó odds ratio (OR) e intervalo de confianza del 95\% (IC) de cada una de las posibles variables predictoras de IBG mediante un modelo bivariado de regresión logística. Luego, se ajustaron las variables predictoras mediante regresión logística múltiple. Nivel estadístico menor a 0,05 . Se calculó la sensibilidad y especificidad del modelo de regresión. El análisis estadístico se realizó utilizando el programa Statistix 8.0. Se compararon las proporciones mediante $\chi^{2}$.

El estudio fue aprobado por el Comité de ética y Docencia e Investigación del Sanatorio Mater Dei.

\section{Resultados}

Se incluyeron en el período estudiado 201 pacientes. El 50,7\% de los pacientes era de sexo femenino. El 34,8\% de los pacientes ingresados al protocolo (n: 70) presentó IBG. En la distribución de los niños según grupo etáreo e IBG, el 73\% (n: 148) tenía $<3$ meses y $37,8 \%$ de éstos (n: 56) ingresó en el grupo con IBG. El 17.9\% (n: 36) de los pacientes correspondió a niños entre 3 y 12 meses, $25 \%$ de éstos (n: 9) presentó IBG. De los 17 niños > 12 meses, 29,4\% (n: 5) desarrolló IBG (Tabla 1).

Se documentaron 72 sitios de infección en los 70 pacientes: infección urinaria (n: 47), meningitis (n: 11), bacteriemia (n: 9), otitis media (n: 4) y parotiditis bacteriana (n: 1). Dos pacientes tuvieron dos sitios diferentes de infección: ambos con infección urinaria y meningitis (uno de ellos con clínica de sepsis). La documentación etiológica de IBG en niños bajo 2 años de edad internados por fiebre sin foco aparente, se señala en la Tabla 2.

\begin{tabular}{|lccc|}
\hline $\begin{array}{l}\text { Tabla 1. Infecciones bacterianas detectadas en } \\
\text { pacientes febriles sin foco, según grupo etáreo }\end{array}$ \\
\hline Edad & $\begin{array}{c}\text { Infección } \\
\text { bacteriana }\end{array}$ & $\begin{array}{c}\text { Resto de } \\
\text { pacientes }\end{array}$ & Total \\
\hline$<3$ meses & 56 & 92 & 148 \\
\hline $3-12$ meses & 9 & 27 & 36 \\
\hline 12 meses & 5 & 12 & 17 \\
\hline $0-24$ meses & 70 & 131 & 201 \\
\hline
\end{tabular}


En los meses de otoño se internaron 42 pacientes, 12 de ellos con IBG, en invierno 47, 16 de ellos con IBG, en primavera 74 pacientes, 25 de ellos con IBG, y en verano 38,17 de ellos con IBG $(\mathrm{p}=0,49)$.

Respecto al número de hermanos, 92 fueron hijos únicos, 71 tenían un hermano, 35 tenían 2 hermanos y 3 tenían 3 hermanos; presentaron IBG: 31, 22, 16 y 1 paciente, respectivamente. El porcentaje de IBG según número de hermanos fue de 33,7\%,31\%, 45,7\% y 33,3\%, respectivamente $(\mathrm{p}=0,5)$

Tabla 2. Etiología de infección bacteriana en niños bajo 2 años de edad internados por fiebre sin foco aparente ( $\mathrm{n}: 72$ infecciones)

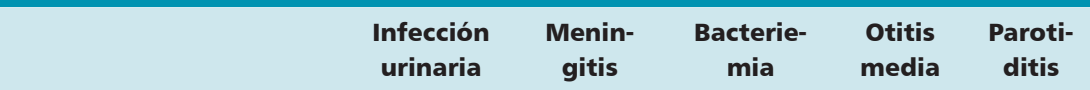

$\begin{array}{lrcc}\text { Escherichia coli } & 35 & 1 & 1 \\ \text { Pseudomonas aeruginosa } & 2 & 1 & 1 \\ \text { Streptococcus agalactiae } & & 1 & 1 \\ \text { Staphylococcus epidermidis } & & 1 & 2\end{array}$

\begin{tabular}{llll|} 
Enterococcus & 3 & 3 & 2 \\
Enterobacter cloacae & 1 & &
\end{tabular}

Klebsiella pneumoniae 6

Streptococcus pneumoniae

Sin documentación etiológica

Total

№ pacientes infectados: 70. Documentación de sitio de infección: 72. Hay 2 pacientes con dos sitios de infección: 2 de ellos con infección urinaria y meningitis.

Tabla 3. Análisis bivariado. Factores de riesgo asociados a infección bacteriana grave

\begin{tabular}{lccc} 
Variable & OR & IC 95\% & p valor \\
Eritrosedimentación $>30 \mathrm{~mm} / \mathrm{h}$ & 2,15 & $1,06-4,35$ & 0,03 \\
\hline Leucocitos $>15.000 / \mathrm{mm}^{3}$ & 2,17 & $1,15-4,10$ & 0,02 \\
\hline Infección viral en el hogar & 0,41 & $0,22-0,75$ & 0,009 \\
Vacunación H. influenzae b & 0,50 & $0,26-0,98$ & 0,04 \\
YIOS (puntaje 1) & 2,7 & $1,15-4,11$ & 0,02 \\
\hline
\end{tabular}

Tabla 4. Análisis multivariado. Factores de riesgo asociados a infección bacteriana grave

\begin{tabular}{lccc|} 
Variable & OR & IC 95\% & p valor \\
\hline Leucocitos $>15.000 / \mathrm{mm}^{3}$ & 2,85 & $1,41-5,76$ & 0,004 \\
\hline Infección viral en el hogar & 0,35 & $0,18-0,67$ & 0,001 \\
\hline
\end{tabular}

Sólo 4,9\% de los niños con IBG (10/201) concurría a jardín maternal $(\mathrm{p}=0,72)$.

De los 201 pacientes, 59,7\% (120/201) presentó criterios de bajo riesgo según Rochester; $83,3 \%$ pertenecían al grupo menores de 3 meses de edad (100/120).

De los 53 niños mayores de 3 meses, 88,6\% había recibido al menos una dosis de vacuna conjugada contra $H$. influenzae tipo b (incluida en el programa nacional), $15,1 \%$ vacuna conjugada heptavalente contra $S$. pneumoniae y $5,7 \%$ vacuna conjugada contra $N$. meningitidis serogrupo $\mathrm{C}$ (ambas opcionales). No se documentaron infecciones por $H$. influenzae tipo b. Se documentaron dos causadas por $S$. pneumoniae.

La mediana de días de internación fue de 4,6 (rango 1-20). El 87,2 \% (n: 129) de los pacientes bajo 3 meses de edad recibió tratamiento antimicrobiano empírico inicial por vía parenteral: cefotaxima + ampicilina (n: 90), ceftriaxona (n: 32), cefotaxima (n: 6) y ampicilina + gentamicina (n: 1). El 69,8\% (n: 37) de los pacientes mayores de 3 meses recibió medicación empírica inicial: ceftriaxona (n: 32) y ampicilina + cefotaxima (n: 5). La duración media de antibioterapia en el lactante bajo 3 meses de edad sin infección documentada fue de 2,4 días.

Los diagnósticos de egreso, fueron: fiebre sin foco documentado (n: 69), infección urinaria (n: 47), infección de vía aérea superior (n: 27), exantema presuntamente viral (n: 13), meningitis (n: 11), gastroenteritis (n: 11), bacteriemia (n: 9), bronquiolitis (n: 8), otitis media aguda (n: 4), parotiditis supurada (n: 1) y sepsis (n: 1). (Total 201)

Las variables predictoras de IBG en el análisis bivariado fueron: recuento de leucocitos $>15.000 / \mathrm{mm}^{3}$, VHS $>30 \mathrm{~mm} / \mathrm{h}$ y como factores protectores: escala de YIOS (puntaje 1 en cada item de la escala), presencia de infección viral en el hogar y antecedentes de vacuna contra $H$. influenzae tipo b. (Tabla 3). En el análisis multivariado las variables asociadas independientemente a IBG fueron: recuento de leucocitos $>$ de $15.000 / \mathrm{mm}^{3}$ como factor de riesgo e infección viral en el hogar como factor protector. (Tabla 4). La especificidad del modelo fue $94 \%$ y la sensibilidad $26 \%$.

\section{Discusión}

El diagnóstico y tratamiento del lactante febril fue y sigue siendo motivo de discusión en la práctica cotidiana ${ }^{3}$. Si bien existen escalas de observación y criterios de riesgo de IBG establecidos, hay variables que no son validadas por todos los estudios o jerarquizadas para la toma de decisiones $^{1,2}$. Por otra parte, no hay gran cantidad de estudios sobre este tema en la población de Latinoamérica ${ }^{11-15}$.

A efectos prácticos, como en otros estudios ${ }^{5,6}$ desarrollaremos la discusión en dos grupos: niños bajo 3 meses de edad y niños entre 3 y 24 meses. 


\section{Niños febriles bajo 3 meses de edad}

Como refiere la bibliografía, en el grupo de pacientes febriles $<60-90$ días, los datos clínicos por sí solos no alcanzan a detectar todos los casos de IBG ${ }^{7,11,16}$. La escala de YIOS de Bonadio para los niños $<8$ semanas es un intento de clasificar a los niños en alto o bajo riesgo de IBG o infección grave $(\mathrm{IG})^{2}$. Ello obliga a buscar otros parámetros de estudios complementarios que ayudaron a diferenciar estos cuadros clínicos. Uno de lo más utilizados son los criterios de bajo riesgo de Rochester que intentan excluir $\mathrm{IBG}^{17,18}$.

Si en la evaluación surgen criterios de alto riesgoaproximadamente en $60 \%$ de los casos referidos por la bibliografía ${ }^{1-3}$ - el paciente debe ser internado, se debe realizar punción lumbar para examen del LCR si no se hizo previamente y medicar con antimicrobianos hasta tener el resultado de los cultivos, ayudando a establecer el diagnóstico con métodos complementarios específicos. Este criterio fue el que nosotros utilizamos en los pacientes internados ${ }^{16}$.

En Buenos Aires, Argentina, en un estudio realizado en 125 lactantes febriles bajo 90 días de vida internados en un hospital pediátrico, se encontraron resultados similares aunque con mayor número de pacientes sin foco documentado ${ }^{11}$.

En nuestro estudio, $87,2 \%$ (n: 129) de los lactantes bajo 3 meses de edad internados recibió tratamiento antimicrobiano empírico inicial. Cabe consignar que aun en este grupo etáreo, la bibliografía en su mayoría considera que la totalidad de los neonatos debe evaluarse para descartar IBG y recibir antibioterapia empírica, hasta disponer del resultado de los cultivos o establecer evidencia de foco $0^{1,7,19,20}$. Nuestra casuística, que incluyó neonatos, fue conformada principalmente por lactantes entre 1 y 3 meses debido a que niños menores se internan habitualmente en la unidad de neonatología.

En el grupo de lactantes de 1 a 3 meses, hay diferentes opciones con relación a medicar o no empíricamente al paciente, pero en general hay consenso que establece que si el paciente presenta alto riesgo de IBG, éste debe ser medicado por lo que debe realizarse la punción lumbar previamente ${ }^{1,5,13,14,21}$. Por otra parte, en el paciente que va a recibir antibióticoterapia es necesaria efectuar antes la punción lumbar ${ }^{4,22}$.

En nuestro estudio se confirma que hubo pacientes de bajo riesgo según los criterios de Rochester que, no obstante, tuvieron IBG. Esto refuerza lo adecuado de considerar la internación en este grupo de pacientes con fiebre sin foco documentado, donde el seguimiento posterior no sea asegurado. Al estar internados y con seguimiento minucioso se pudo documentar con posterioridad focos de infección (otitis media, infección urinaria, parotiditis supurada).

Por otra parte, la presencia de infección viral en el hogar como factor protector de IBG en el análisis multivariado representa un elemento novedoso para evaluar mayores o menores estudios, aun en el paciente que fue internado para observación.

\section{Niños febriles entre 3 y 24 meses}

El riesgo de bacteriemia oculta en niños entre 3 y 24 ó 36 meses de edad con fiebre sin foco aparente, oscila entre 3 y $11 \%$ con una probabilidad media de $4,3 \%$ en pacientes en los que se documenta fiebre mayor de 39 ${ }^{\circ} \mathrm{C}^{24-26}$. "El riesgo de desarrollar una meningitis en pacientes no vacunados contra $\mathrm{H}$. influenzae tipo b en el curso de bacteriemias causadas por $S$. pneumoniae, $H$. influenzae tipo b y $N$. meningitidis es de $3 \%$, (28,29), $15 \%$ y $58 \%$, respectivamente. El riesgo de bacteriemia disminuyó considerablemente con la introducción de la vacuna contra $H$. influenzae tipo b en el Programa Nacional de Inmunizaciones de Argentina en 1998. (Fuente: Sistema Nacional de Vigilancia Epidemiológica. Ministerio de Salud de la República Argentina). La cobertura de vacuna contra $H$. influenzae tipo b de $88,6 \%$ en nuestro estudio es similar a la cobertura de vacuna cuádruple (H. influenzae tipo b, difteria, tétanos y tos convulsiva) de los residentes de la Ciudad de Buenos Aires del año 2004 (Fuente: Ministerio de Salud de la República Argentina) ${ }^{29}$. Otros agentes infecciosos relacionados con bacteriemia oculta son Staphylococcus aureus, Streptococcus pyogenes y Salmonella sp. El porcentaje de 4,5\% de bacteriemias en nuestro estudio es inferior a otro estudio argentino de niños de 1 a 24 meses. No obstante, en aquel estudio se tomaron todos los niños febriles, con o sin foco aparente de infección ${ }^{30}$.

Sin que el paciente presente aspecto tóxico, la consideración del diagnóstico de infección urinaria en el lactante es importante ${ }^{31}$. Se calcula que esto ocurre en $5 \%$ de los pacientes sobre 3 meses de edad concurrentes con fiebre a una unidad de emergencia. La temperatura $>$ $39^{\circ} \mathrm{C}$ predispone a un mayor riesgo de infección urinaria. Un recuento de $>10$ leucocitos/campo se encuentra en $90 \%$ de las infecciones urinarias en este grupo etáreo. Un sedimento urinario normal, por otra parte, no descarta el diagnóstico, por lo que el urocultivo es el método diagnóstico de elección. Debemos reiterar que el diagnóstico de infección urinaria no se realizó al ingreso sino con posterioridad a su internación.

Una cuidadosa elección del paciente en quien no se presuma el diagnóstico de infección viral, con persistencia de la fiebre mayor de $39^{\circ} \mathrm{C}$ y su posible seguimiento en forma ambulatoria con controles frecuentes, es una alternativa válida para el diagnóstico correcto ${ }^{3,11}$. En el grupo etáreo de 3 a 24 meses donde la frecuencia de cuadros virales se presume mayor, se internaron 53 . El consenso para el abordaje del paciente con fiebre sin foco en este grupo etáreo no es uniforme ${ }^{10,16,32,33}$. Excede al objetivo 
de este trabajo considerar las causas que llevaron al pediatra de cabecera o al médico de urgencias a internar al paciente; en ocasiones se decidió su internación sin estudios invasores $\mathrm{u}$ otros exámenes de laboratorio. De todos modos para que estos pacientes hubieran sido incluidos en nuestro estudio, no debían tener foco clínico evidente de infección al ingreso.

La cuestión se centra entonces en cómo encarar la terapéutica. Es conveniente evitar la utilización de antimicrobianos en el paciente que impresiona en buen estado general, habitualmente seguido en forma ambulatoria ${ }^{34-36}$. De los 53 pacientes internados con 3 o más meses de edad, en 17 se documentó IBG. De los que no tuvieron documentación de IBG, 69,4\% no presentó recuento de leucocitos $>15.000 / \mathrm{mm}^{3}$. De los 17 que tuvieron IBG, hubo 4 que tuvieron recuento de leucocitos $\leq 15.000 / \mathrm{mm}^{3}$; en ellos se documentó finalmente una infección urinaria y hubo 2 pacientes con recuento de leucocitos $\leq 15.000 /$ $\mathrm{mm}^{3}$ cuyo diagnóstico de egreso fue otitis media aguda. Algunos de estos pacientes pudieron haber sido evaluados en el contexto de unidades de urgencias sin necesidad de internación. Si exclusivamente hubiera sido considerado el recuento de leucocitos $>15.000 / \mathrm{mm}^{3}$ como riesgo para medicar empíricamente al paciente, se habrían tratado 5 de las 9 infecciones urinarias documentadas en el niño mayor de 3 meses, probablemente por vía intravenosa. Las otras cuatro hubieran sido tratadas 24 a 48 horas después, probablemente por vía oral, sin necesidad que ello implicara un peor pronóstico del paciente. De las 4 otitis media documentadas en la internación, dos hubieran sido medicadas considerando el recuento de leucocitos mayor de $15.000 / \mathrm{mm}^{3}$, y las otras dos hubieran sido medicadas con posterioridad al diagnóstico clínico, también en una patología con baja posibilidad de bacteriemia y complicaciones.

En síntesis: si en el futuro consideráramos tratar al paciente mayor de 3 meses internado por fiebre sin foco aparente, según factores de riesgo de nuestro estudio, $58,5 \%$ no hubieran recibido antimicrobianos en forma empírica, contra el $69,8 \%$ que si los recibió. Esto disminuiría la utilización inadecuada de antimicrobianos ${ }^{35,37-39}$, la generación de resistencia a los mismos y mayor tiempo de hospitalización. En nuestro trabajo, las 4 bacteriemias documentadas en los niños mayores de 3 meses, (2 por $S$. pneumoniae, 1 por Enterococcus y 1 por Pseudomonas aeruginosa) hubieran recibido tratamiento empírico inicial por criterio de riesgo. Con la vacuna conjugada heptavalente, que recibió sólo el $15 \%$ de los pacientes que requirieron internación, la posibilidad de disminuir las infecciones por este agente no fue tan evidente. La vacuna antineumocóccica conjugada heptavalente disponible en ese momento, tiene en la Argentina cobertura sólo para $45 \%$ de las meningitis por este agente y $62,6 \%$ de la enfermedad invasora en niños menores de 2 años $^{40}$.
Los dos pacientes en quienes se documentó bacteriemia por S. pneumoniae fueron detectados por los criterios de alto riesgo para infección bacteriana en nuestro estudio. Uno de ellos estaba vacunado y no se realizó identificación serológica de la cepa recuperada y el otro no había recibido la vacuna. No disponiendo en nuestro país y en forma universal una vacuna adecuada con elevada cobertura contra los serotipos prevalentes causante de enfermedad invasora por $S$. pneumoniae $e^{41}$, la utilización del recuento de leucocitos $\geq 15.000 / \mathrm{mm}^{3}$ permite acercarse a conductas mas adecuadas evitando el riesgo de IBG en el paciente observado en servicios de urgencia o eventualmente internados ${ }^{10}$. En nuestro estudio este punto de corte refuerza la probabilidad de mayor documentación de IBG en estos pacientes. Esta hipótesis podría modificarse, como lo señala la bibliografía, si revaloramos la conducta en el paciente mayor de 3 meses y menor de 24 meses, con buen aspecto general, con realización de sedimento urinario y urocultivo en la unidad de emergencia y ponderado el antecedente de vacunación con dos ó más dosis de vacuna anti-neumocóccica conjugada con 10 ó 13 serotipos $^{5,42,43}$.

En este estudio encontramos que el recuento de leucocitos mayor a $15.000 / \mathrm{mm}^{3}$ aumenta 2,85 veces el riesgo de IBG y la presencia de infección viral en el hogar disminuye 0,35 veces el riesgo de IGB.

Limitaciones de nuestro estudio radican en el número total de pacientes incluidos y el hecho que se trató sólo de pacientes internados, lo que puede aumentar la proporción de IBG. No obstante, el seguimiento de los mismos en forma minuciosa en los aspectos clínicos y laboratorio nos permitió la detección de IBG aun en aquellos pacientes inicialmente considerados de bajo riesgo.

\section{Conclusiones}

En nuestro medio y en este grupo etáreo, $36 \%$ de los niños internados por fiebre sin foco presentó infección bacteriana grave. El factor asociado a desarrollar infección bacteriana grave fue el recuento de leucocitos mayor de $15.000 / \mathrm{mm}^{3}$. La presencia de infección viral en el hogar demostró ser un factor de protección, disminuyendo casi en $80 \%$ el riesgo de tener una infección bacteriana grave.

\section{Resumen}

Objetivo: Analizar los factores de riesgo asociados a infección bacteriana grave (IBG) en niños $<2$ años internados por fiebre sin foco aparente. Población: Niños bajo 2 años de edad internados en el Servicio de Pediatría del Sanatorio Mater Dei entre mayo 2004 y diciembre 2005. Diseño: Observacional, prospectivo, longitudinal. Material y Métodos: Variables independientes: edad, 
sexo, hermanos, infección viral en el hogar, concurrencia a jardín maternal, vacunación, época del año, laboratorio, criterios de Rochester y escala de YIOS, tratamiento antimicrobiano, días de internación y diagnóstico final. Se utilizó un modelo de regresión logística múltiple por pasos. Resultados: Setenta IBG de 201 pacientes, 56 $<3$ meses de edad. Diagnósticos principales: infección urinaria (n: 47), meningitis (n: 11), bacteriemias (n: 9). Factores predictores: recuento de leucocitos $>$ de 15.000/ $\mathrm{mm}^{3}$ (coef. 1,05 OR: 2,17 IC 1,13-4,15) e infección viral en el hogar (coef. -0,79 OR 0,42, IC 0,23-0,77). Conclusiones: El factor asociado a IBG fue recuento de leucocitos $>15.000 / \mathrm{mm}^{3}$. La presencia de infección viral en el hogar demostró ser un factor de protección.

\section{Referencias}

1.- Jaskiewicz J A, Mc Carthy C A, Richardson A M, White K C, Jisha D J, Dagan R. Lactantes con fiebre expuestos a un bajo riesgo de infección bacteriana grave. Valoración de los criterios de Rochester e implicaciones para el tratamiento. Pediatrics (ed. esp.) 1994; 38 : 169-74.

2.- Bonadio W A. The history and physical assessment of the febrile infant. Pediatr Clin North Am 1998; 45: 65-77.

3.- Baker M D, Bell L M, Avner J R. Outpatients management without antibiotics of fever in selected infants. N Engl J Med 1993; 329: 1437-41.

4.- Baskin M N. The prevalence of serious bacterial infections by age in febrile infants during the first 3 months of life. Pediatr Ann 1993; 22: 462.

5.- Baraff L J. Management of infants and young children with fever without source. Pediatr Ann 2008; 37: 673-9.

6.- Ishimine P. Fever without source in children 0 to 36 months of age. Pediatr Clin North Am 2006; 53: 167-94.

7.- Baker M D, Bell L M. Unpredictability of serious bacterial illness in febrile infants from birth to 1 month of age. Arch Pediatr Adolesc Med 1999; 153: 508-11.

8.- American College of Emergency Physicians Clinical Policies Committee. Clinical Policy for children younger than three years presenting to the emergency department with fever. Ann Emerg Med 2003; 42: 530-45.

9.- Finkelstein J A, Christiansen C L, Platt R. Fever in pediatric primary cares: occurrence, management and outcomes. Pediatrics 2000; 105: 260-6.

10.- Baraff L J, Bass J W, Fleisher G R, Klein J O, Mc Cracken G H Jr, Powell K R, et al. Practice guideline for the management of infants and children 0 to 36 months of age with fever without source. Agency for Health Care Policy and Research. Ann Emerg Med 1993; 22: 1198-210.

11.- Ellis A, Onaindia J, Berenstein A, Freidzon E, Cazés C, Awad A. Lactantes febriles menores de 90 días, internados en un hospital pediátrico. Rev Hosp Niños Buenos Aires 1994; XXXVI: 312-24.
12.- Ibarra X, Viviani S T, Peña A M, Cerdá J. Síndrome febril sin foco y sospecha de infección bacteriana en niños entre 6 semanas y 36 meses. Rev Chil Pediatr 2008; 79: 388-92.

13.- Diaz Á M, Viel R Y, Acosta B B, Claver I D. ¿Es necesaria la punción lumbar en la evaluación del recién nacido febril sin signos de focalización? Rev Chil Infect 2008; 25: 179-183.

14.- Marcondes Machado B, Morais Cardoso D, de Paulis M, Ulhóa Escobar A M, Elias Gilio A. Fever without source: evaluation of a guideline. J Pediatr (Río J) 2009; 85: 426-32.

15.- Brockmann V P, Ibarra G X, Silva W I, Hirsh B T. Etiología del síndrome febril sin foco en niños bajo 36 meses de edad que consultan a un servicio de urgencia. Rev Chil Infect 2007; 24: 33-9.

16.- Baker M D, Bell L M, Avner J R. The efficacy of routine outpatient management without antibiotics of fever in selected infants. Pediatrics 1999; 103: 627-31.

17.- Dagan R, Powell K R, Hall C B, Menegus M A. Identification of infants unlikely to have serious bacterial infection although hospitalized for suspected sepsis. J Pediatr 1985; 107: 855-60.

18.- Dagan R, Sofer D, Phillip M. Ambulatory care of febrile infants younger than 2 months of age classified as being at low risk for having serious bacterial infections. J Pediatr 1988; 112: 355-60.

19.- Ferrera P C, Bartfield J M, Snyder H S. Neonatal fever: Utility of the Rochester criteria in determining low risk for serious bacterial infection. Am J Emerg Med 1997; 15: 299-302.

20.- Kadish H A, Loveridge B,Tobey J, Bolte R G, Corneli H M. Applying outpatient protocols in febrile infants 1-28 days of age: Can the threshold be lowered? Clin Pediatr (Phila) 2000; 39: 81-8.

21.- Bonsu B K, Harper M B. Utility of the peripheral white blood cell count for identifying sick young infants who need lumbar puncture. Ann Emerg Med 2003; 41: 206-14.

22.- Meehan W P, Bachur R G. Predictors of cerebrospinal fluid pleocytosis in febril infants aged 0 to 90 days. Pediatr Emerg Care 2008; 24: 287-93.

23.- Baraff L J. Management of fever without source in infants and children. Ann Emerg Med 2000; 36: 602 .
24.- Kuppermann N, Fleisher G, Jaffe D. Predictors of occult pneumococcal bacteremia in young febrile children. Ann Emerg Med 1998; 31: 679-87.

25.- Mc Carthy P L, Sharpe M R, Spiesel S Z, Dolan T F, Forsyth B W, Dewill T G, et al. Observation scales to identify serious illness in febrile children. Pediatrics 1992; 70: 802-9.

26.- International Pediatrics Consensus Conference: Definition for sepsis and organ dysfunction in pediatrics. Pediatr Crit Care Med 2005; 6: 2-8.

27.- Shapiro E D, Aaron N H, Wald E R. Risk factors for development of bacterial bacterial meningitis among children with occult bacteremia. J Pediatr 1986; 109: 15-19.

28.- Harper M B, Bachur R, Fleisher G R. Effect of antibiotic therapy on the outcome of outpatients with unsuspected bacteremia. Pediatr Infect Dis J 1995; 14: 760-7.

29.- Dayan G, Orellana L, Forlenza R, Ellis A, Chaui J, Kaplan S, et al. Vaccination coverage among children aged 13 to 59 months in Buenos Aires, Argentina, 2002. Pan J Public Health 2004; 16: 158-67.

30.- Straface R. El niño febril de 1 a 24 meses de edad. Arch. Argent. Pediatr 2001; 99: 1-18.

31.- Zorc J J, Levine D A, Platt S L, Dagan P S, Macias C G, Krief W. Clinical and demographic factors associated with urinary tract infection in young febrile infants. Pediatrics $2005 ; 116$ : 644.

32.- Greens D S, Harper M B. Low risk of bacteremia in febrile children with recognizable viral syndromes. Pediatr Infect Dis J 1999; 18: 258-61.

33.- Krishnan S. Evaluation and management of the febrile infant in the emergency department. Indian J Pediatr 2003; 70 Suppl 1:S45-50.

34.- Baker M D. Evaluation and management of infants with fever. Pediatr Clin North Am 1999; 46: 1061-72.

35.- Pantell R H, Newman T B, Bernzweig J, Bergman D A, Takayama J I, Segal M. Management and outcomes of care of fever in early infancy. JAMA 2004; 291: 1203-12.

36.- Comité Nacional de Infectología. Sociedad Argentina de Pediatría. Lactante febril menor de 36 meses. Libro Azul de Infectología Pediátrica, $2^{\mathrm{a}}$ edición, Buenos Aires, Editorial Ideográfica, 2001; 139-45. 
37.- Jain S, Sullivan K. Ceftriaxone use in the emergency department: Are we doing it right? Pediatr Emerg Care 2002; 18: 259-64.

38.- Stamos J K, Shulman S T. Abandoning empirical antibiotics for febrile children. Lancet 1997; 350:84.

39.- Quinteros M, Radice M, Famiglieti A, y SIR. Análisis de la sensibilidad a los antimicrobianos en los aislamientos de pacientes internados. Años 2004-2005. Asociación Argentina de Microbiología 2006. Boletín No 172; 13-6.

40.- Ruvinsky R. Epidemiología de las infecciones invasoras por Streptococcus pneumoniae en la Argentina: Un sistema de vigilancia, 1993-2004. Rev. Hosp. Niños Bs. Aires 2005; 47: 222-32.

41.- Ellis A, Fossati S, Efron A, Garea M, Vay C, García Roig C, et al. Epidemiological report of invasive pneumococcal disease in a private clinic of Buenos Aires. World Society for Pediatric Infect Dis Congress. Buenos Aires, Argentina. Nov 2009; Abstract Book, pág 358 .

42.- Capapé Zache S, Luaces Cubells C,
Garrido Romero R, Claret Tertuel G,

Fernández Landaluce A, Benito Fernández J. Impacto de la vacunación neumocócica en el manejo del lactante con fiebre, en relación al porcentaje de vacunación. An Pediatr (Barc) 2007; 67: 30-6.

43.- Mintegui S, Benito J, González M, Astobiza E, Sánchez J, Santiago M. Impact of pneumococcal conjugate vaccine in the management of highly febrile children aged 6 to 24 months in an emergency department. Pediatr Emerg Care 2006; 22: 566-9. 